\title{
Motion to Reduce Weight and Body Shape
}

\author{
Jian Liu, Lijun Xu \\ Northeast DianLi University School of Physical Education Jilin City Jilin Province China \\ 20009971@qq.com
}

\begin{abstract}
Through the analysis principle of reason and exercise on obese produce weight loss, put forward the movement to lose weight and the method of scientific health, advocate the beautiful shape of the body through exercise, ang achieve healthy and beautiful goal.
\end{abstract}

\section{Keywords-Obesity exercise form}

Obesity was predicted to be the first killer of the twentyfirst Century human health pandemic. Modern medicine has proved, that obesity is easier to cause disease than 20 species. Obesity not only affects the health but changes in posture, bloated body formation. Scientific and proper exercise and proper nutrition, can enhance physical fitness, improving body function, reduce body fat and create a healthy, beautiful shape, to achieve a body purpose of weighting loss.

\section{THE EXERCISE TO LOSE WeIGHT}

\section{A. Criteria for obesity}

Who defines obesity as excessive fat accumulation caused by chronic and non-communicable diseases and health damage in the body.

Body mass index BIM evaluation: the weight is divided into ideal weight, overweight and obesity. Method: BMI = body weight $(\mathrm{kg}) /$ height $(\mathrm{m})$. BMI in $18.5 \sim 24.9$ belongs to the normal range; $>25$ is $25 \sim 29.5$ for the obesity; $30 \sim$ 34.9 level of obesity; $35 \sim 39.9$ to two obese; $\geq 40$ grade three obesity. Our Oriental $>28$ is for obesity. The fat content of evaluation: we put the body into the lean body mass and fat part, expressed as a percentage of body weight which each accounted for. Lean body part includes all the tissues and organs. in addition to fat Fat including essential fatty acids and the body of the "extra" fat. The normal value of body fat content: men should be in $10 \sim 20$, women should be in $15 \sim 25$; girth evaluation: through the waist to hip ratio index (waist / hip) to the evaluation of abdominal fat distribution standard, male female $>0.8>1$;

\section{B. Reason of obesity}

Obesity is caused by a variety of factors. In addition to genetic, endocrine disorders, energy balance is the key to weight. Energy intake and consumption equal, energy balance, the weight will not change; energy intake is greater than the energy consumption, energy balance and weight gain; energy intake is less than the energy consumption, energy balance, weight reducing. Energy intake is obtained through the diet, consumption of energy is consumed in the maintenance of normal body state of life, work, exercise of energy. Intake of too much energy will be converted into fat, and the fat stored in the body.Body fat content in vivo is increasing, and over time will form the accumulation of fat, leading to obesity.

\section{The principle of exercise for weight loss}

Exercise can increase body fat and blood fat consumption, reduce the occurrence of obesity. We can lose weight through activities such as walking, running or swimming in the consumption of energy. When increasing the consumption of energy and movement without extra calories and fat negative balance is not the supplementary motor energy consumption caused by energy .It can make the body weight or body fat reduction. So we can increase on the sports consumption to control the energy balance and reduce redundant adipose, achieve the goal of weight loss. Previous, exercise for weight loss methods are varied. You can choose the methods according to their own conditions and environment etc. The time and frequency of the movement, usually 30 to 60 minutes, three times a week. Exercise intensity should be $50 \sim 75 \%$ of the maximum oxygen consumption and heart rate control in maximum heart rate $(220$ - age) x $60 \sim 80 \%$. Strength of not less than the maximum heart rate of $60 \%$ is also called intensity threshold. Sources of sugar in blood and liver on this premise is consumed, so fat can be used, and obtain the sufficient combustion. Characteristics of lipid metabolism in sports are to mobilize the slower, usually peak in 2-4 hours late; and long time movement reached 90\%.

\section{II.SHAPES}

\section{A. The shape is relatively stable characteristics acquired based on genetic variation}

It mainly reflects the external features of the body, including the bones, muscles etc.. Congenital genetic determines the structure of bone, such as the shoulder width, upper and lower limbs ratio. At the same time, if you acquired scientific training will also have a good effect on the body such as the use of muscles to increase the shoulder width. It can make the buttocks muscles up to improve the breech line. 
The man requires the muscular body to be well proportioned, vigorous and masculine, and the woman requires clear lines, body symmetry, flexible integration, and reflects the female physiological and anatomical characteristics of women embody all curve beauty of symmetry, harmony and beauty of elasticity.

The motion controls balance of body fat and energy of the body, but also can develop muscle through the local muscle exercise, increasing the surrounding muscles, and improving the body of imperfect factors, and shaping beauty. For example, man's shoulder is not wide enough, the chest and the back is not thick, and the body slightly is thin. These deltoid, pectoralis major muscle, trapezius, latissimus dorsal exercises and other large muscle groups and collaborative small muscles can be developed. And women may shape curve through the development of the chest and back, abdomen, and gluteus maximus. At the same time through the physique training control body posture, the body symmetry and harmonious can be developed.

1) Methods of various parts development of the body muscles

Grasp the strength, frequency, and velocity can develop the body muscles. Intensity refers to the weight of a group or an exercise; density refers to the time between the group and group. High strength: my greatest strength of $90 \sim$ $100 \%$ times, corresponding to $1 \sim 3,1 \sim 2,2 \sim 3$ minutes interval time, slow speed; in my strength, maximum strength $75 \sim 85 \%$ times, corresponding to $6,8,10,12,4 \sim$ 6 group, intermittent time $30 \sim 60$ second, in the small and slow speed; strength: my greatest strength $70 \%$, more than 16 times, $4 \sim 6$ group, intermittent time can be short or long, and mainly the fast speed. The developed muscle program is the first major muscle group after the small muscles; flexor extensor first after the first leg and arm; then developed the waist.

\section{Body training principle \\ 2) Comprehensive}

a)

The human body is an organic whole, and each organ system function is the organic connections. Development and improving the function of each part of the system depend on the other part of the system to improve the performance of each. So we should deal with the relationship between local and global part. Use a variety of methods, contents and methods, so that all parts of the body can obtain the development.

b) Proceed from the reality

Start from their own physical condition and the objective external reality. Scientifically select the practice content, means, and methods to develop targeted exercises.

c) Step by step

With the objectives, contents and methods selection, the load arrangement must be from easy to difficult, from simple to complex, from the shallower to the deeper, from small to big, and gradually improve; uninterrupted: adhere to the continuous, systematic and continuous scientific training. Long term unremittingly and maintaining consistency can receive the good effect.

Long time aerobic exercise can lose excess body fat, and make weight loss; local muscle exercises to create a beautiful shape. These two methods must be organically combined. Simply losing weight will form a "big turnip into small radish", so through a comprehensive and scientific exercising can lose fat and shape the perfect body.

\section{REFERENCES}

[1] Zhu peibang. Exercise to lose weight principle. Journal of Ningxia Teachers' University [J].2007-05 -15

[2] Meng Xia. Talk about exercise to lose weight from the human body energy metabolism. Science and technology information (Research) [J].2007-09-15 